\title{
Correction
}

\section{Correction: Kim, Tsai et al., “Attention to Multiple Objects Facilitates Their Integration in Prefrontal and Parietal Cortex"}

In the article "Attention to Multiple Objects Facilitates Their Integration in Prefrontal and Parietal Cortex" by Yee-Joon Kim, Jeffrey J. Tsai, Jeffrey Ojemann, and Preeti Verghese, which appeared on pages 4942-4953 of the May 10, 2017 issue, an incorrect affiliation for author Yee-Joon Kim was listed. The authors apologize for the oversight. His corrected affiliation is as follows: Center for Cognition and Sociality, Institute for Basic Science, Daejeon 34141, Republic of Korea and Smith-Kettlewell Eye Research Institute, 2318 Fillmore Street, San Francisco, CA 94115. In addition, the corrected affiliation for correspondence regarding the article is as follows: Correspondence should be addressed to Dr. Yee-Joon Kim, Center for Cognition and Sociality, Institute for Basic Science, Daejeon 34141, Republic of Korea. E-mail: joon@ibs.re.kr. This correction does not affect the conclusions of the paper. The author line has been corrected on the online PDF version.

DOI:10.1523/JNEUROSCI.0886-18.2018 